\title{
The Lagrangian and Hamiltonian analysis of some relativistic electrodynamics models and their quantization
}

\author{
N.N. Bogolubov (Jr. $)^{* 1,2}$, A.K. Prykarpatsky $y^{\dagger 3,4}$ \\ 1 The V.A. Steklov Mathematical Institute of RAN, Moscow, Russian Federation \\ 2 The Abdus Salam International Centre for Theoretical Physics, Trieste, Italy \\ 3 The AGH University of Science and Technology, Kraków 30-059, Poland \\ 4 The Ivan Franko State Pedagogical University, Drohobych, Lviv region, Ukraine
}

Received June 19, 2009, in final form July 15, 2009

The work is devoted to the study of the Lagrangian and Hamiltonian properties of some relativistic electrodynamics models and is a continuation of our previous investigations. Based on the vacuum field theory approach, the Lagrangian and Hamiltonian reformulation of some classical electrodynamics models is devised. The Dirac type quantization procedure, based on the canonical Hamiltonian formulation, is developed. Within the approach proposed in the work a possibility of the combined description both of electrodynamics and gravity is analyzed.

Key words: Lagrangian and Hamiltonian formalism, relativistic electrodynamics models, Dirac type quantization

PACS: $11.10 . W x, 05.30 .-d$

\section{Introduction}

The classical relativistic electrodynamics of a freely moving charged point particle in the Minkovski space-time $\mathbb{M}^{4}:=\mathbb{E}^{3} \times \mathbb{R}$ is, as well known, based $[5,9,11,31]$ on the Lagrangian formalism assigning to it the following Lagrangian function

$$
\mathcal{L}:=-m_{0}\left(1-u^{2}\right)^{1 / 2}
$$

where $m_{0} \in \mathbb{R}$ is the so-called particle rest mass and $u \in \mathbb{E}^{3}$ is its spatial velocity in the Euclidean space $\mathbb{E}^{3}$, expressed here and throughout further in the light speed units (that is the light speed $c$ units). The least action Fermat principle in the form

$$
\delta S=0, \quad S:=-\int_{t_{1}}^{t_{2}} m_{0}\left(1-u^{2}\right)^{1 / 2} \mathrm{~d} t
$$

for any fixed temporal interval $\left[t_{1}, t_{2}\right] \subset \mathbb{R}$ gives rise to the well known relativistic relationships for the mass of the particle

$$
m=m_{0}\left(1-u^{2}\right)^{-1 / 2}
$$

the momentum of the particle

$$
p:=m u=m_{0} u\left(1-u^{2}\right)^{-1 / 2}
$$

\footnotetext{
*E-mail: nikolai_bogolubov@hotmail.com

†E-mail: pryk.anat@ua.fm, prykanat@cybergal.com
} 
and the energy of the particle

$$
\mathcal{E}_{0}=m=m_{0}\left(1-u^{2}\right)^{-1 / 2} .
$$

The origin of Lagrangian (1), owing to the reasonings from [11,31], can be extracted from the action expression

$$
S:=-\int_{t_{1}}^{t_{2}} m_{0}\left(1-u^{2}\right)^{1 / 2} \mathrm{~d} t=-\int_{\tau_{1}}^{\tau_{2}} m_{0} \mathrm{~d} \tau,
$$

on the suitable temporal interval $\left[\tau_{1}, \tau_{2}\right] \subset \mathbb{R}$, where, by definition,

$$
\mathrm{d} \tau:=\mathrm{d} t\left(1-u^{2}\right)^{1 / 2}
$$

and $\tau \in \mathbb{R}$ is the so-called proper temporal parameter assigned to a freely moving particle with respect to the "rest" reference system $\mathcal{K}_{\mathrm{r}}$. The action (6) looks a bit controversial from the dynamical point of view, since it is physically defined with respect to the "rest" reference system $\mathcal{K}_{\mathrm{r}}$, giving rise to the constant action $S=-m_{0}\left(\tau_{2}-\tau_{1}\right)$, as limits of integrations $\tau_{1}<\tau_{2} \in \mathbb{R}$ were taken to be fixed from the very beginning and no other constraints were imposed. Moreover, considering this particle as charged with a charge $q \in \mathbb{R}$ and moving in the Minkovski space-time $\mathbb{M}^{4}$ under the action of an electromagnetic field $(\varphi, A) \in \mathbb{R} \times \mathbb{E}^{3}$, the corresponding classical (relativistic) action functional is chosen (see $[5,9,11,31])$ as follows:

$$
S:=\int_{\tau_{1}}^{\tau_{2}}\left[-m_{0} \mathrm{~d} \tau+q\langle A, \dot{r}\rangle \mathrm{d} \tau-q \varphi\left(1-u^{2}\right)^{-1 / 2} \mathrm{~d} \tau\right],
$$

with respect to the so-called "rest" reference system, parameterized by the Euclidean space-time variables $(r, \tau) \in \mathbb{E}^{4}$, where as before, $\langle\cdot, \cdot\rangle$ is the standard scalar product in the related Euclidean subspace $\mathbb{E}^{3}$ and there is denoted $\dot{r}:=\mathrm{d} r / \mathrm{d} \tau$ in contrast to the definition $u:=\mathrm{d} r / \mathrm{d} t$. The action (8) can be rewritten as follows, with respect to the reference system moving with velocity vector $u \in \mathbb{E}^{3}$

$$
S=\int_{t_{1}}^{t_{2}} \mathcal{L} \mathrm{d} t, \quad \mathcal{L}:=-m_{0}\left(1-u^{2}\right)^{1 / 2}+q\langle A, u\rangle-q \varphi,
$$

on the suitable temporal interval $\left[t_{1}, t_{2}\right] \subset \mathbb{R}$, giving rise to the following $[5,9,11,31]$ dynamical expressions

$$
P=p+q A, \quad p=m u
$$

for the particle momentum and

$$
\mathcal{E}_{0}=\left[m_{0}^{2}+(P-q A)^{2}\right]^{1 / 2}+q \varphi
$$

for the particle energy, where, by definition, $P \in \mathbb{E}^{3}$ means the common momentum of the particle and the ambient electromagnetic field at a space-time point $(r, t) \in \mathbb{M}^{4}$.

The obtained expression (11) for the particle energy $\mathcal{E}_{0}$ also looks a bit controversial, since the potential energy $q \varphi$, entering additively, has no impact onto the particle mass $m=m_{0}\left(1-u^{2}\right)^{-1 / 2}$. As it was already mentioned [14] by L. Brillouin, the fact that the potential energy has no impact on the particle mass tells us that "... any possibility of the existence of the particle mass related with an external potential energy, is completely excluded". This and some other special relativity theory and electrodynamics problems, as is well known, urged many other prominent physicists of the past $[4,14,19,31,32]$ and of the present $[18,20-24,27-30,33,34,37,38]$ to make significant efforts aiming to develop alternative relativity theories based on completely different space-time and matter structure principles.

There also is another controversial inference from the action expression (9). As one can easily show $[5,9,11,31]$, the corresponding dynamical equation for the Lorentz force is given as follows:

$$
\mathrm{d} p / \mathrm{d} t=F:=q E+q u \times B,
$$


where the operation " $\times$ " denotes, as before, the standard vector product and we put, by definition,

$$
E:=-\partial A / \partial t-\nabla \varphi
$$

for the related electric field and

$$
B:=\nabla \times A
$$

for the related magnetic field, acting on the charged point particle $q$; the operation " $\nabla$ " is here, as before, the standard gradient. The obtained expression (12) means, in particular, that the Lorentz force $F$ depends linearly on the particle velocity vector $u \in \mathbb{E}^{3}$, giving rise to its strong dependence on the reference system with respect to which the charged particle $q$ moves. Namely, the attempts to reconcile this and some related controversies $[4,14,18,26]$ forced $A$. Einstein to devise his special relativity theory and proceed further to creating his general relativity theory trying to explain the gravity by means of a geometrization of space-time and matter in the Universe. Here we must mention that the classical Lagrangian function $\mathcal{L}$ in $(9)$ is written by means of the mixed combinations of terms expressed by means of both the Euclidean "rest" reference system variables $(r, \tau) \in \mathbb{E}^{4}$ and an arbitrarily chosen reference system variables $(r, t) \in \mathbb{M}^{4}$.

These problems were recently analyzed from a completely another "no-geometry" point of view in $[6,7,18]$, where new dynamical equations were derived, being free of the controversy mentioned above. Moreover, the devised approach allowed to avoid the introduction of the well known Lorentz transformations of the space-time reference systems with respect to which the action functional (9) is invariant. From this point of view there are very interesting reasonings of work [22], in which there are reanalyzed Galilean invariant Lagrangians, possessing the intrinsic Poincare-Lorentz group symmetry. Below we will reanalyse the results obtained in [6,7] from the classical Lagrangian and Hamiltonian formalisms, which will shed a new light on the related physical backgrounds of the vacuum field theory approach to the common study of electromagnetic and gravitational effects.

\section{The vacuum field theory electrodynamics equations: Lagrangian analysis}

\subsection{A freely moving point particle - an alternative electrodynamical model}

Within the vacuum field theory approach to the common description of the electromagnetism and the gravity, devised in $[6,7]$, the main vacuum potential field function $\bar{W}: \mathbb{M}^{4} \rightarrow \mathbb{R}$, related to a charged point particle $q$, satisfies, in the case of the "rested" external charged point objects, the following [6] dynamical equation

$$
\frac{\mathrm{d}}{\mathrm{d} t}(-\bar{W} u)=-\nabla \bar{W}
$$

where, as above, $u:=\mathrm{d} r / \mathrm{d} t$ is the particle velocity with respect to some reference system.

To analyse the dynamical equation (15) from the Lagrangian point of view we will write the corresponding action functional as

$$
S:=-\int_{t_{1}}^{t_{2}} \bar{W} \mathrm{~d} t=-\int_{\tau_{1}}^{\tau_{2}} \bar{W}\left(1+\dot{r}^{2}\right)^{1 / 2} \mathrm{~d} \tau,
$$

expressed with respect to the "rest" reference system $\mathcal{K}_{\mathrm{r}}$. Having fixed proper temporal parameters $\tau_{1}<\tau_{2} \in \mathbb{R}$, from the least action condition $\delta S=0$ one easily finds that

$$
p:=\partial \mathcal{L} / \partial \dot{r}=-\bar{W} \dot{r}\left(1+\dot{r}^{2}\right)^{-1 / 2}=-\bar{W} u, \quad \dot{p}:=\mathrm{d} p / \mathrm{d} \tau=\partial \mathcal{L} / \partial r=-\nabla \bar{W}\left(1+\dot{r}^{2}\right)^{1 / 2},
$$

where, owing to (16), the corresponding Lagrangian function

$$
\mathcal{L}:=-\bar{W}\left(1+\dot{r}^{2}\right)^{1 / 2} .
$$


Recalling now the definition of the particle mass

$$
m:=-\bar{W}
$$

and the relationships

$$
\mathrm{d} \tau=\mathrm{d} t\left(1-u^{2}\right)^{1 / 2}, \quad \dot{r} \mathrm{~d} \tau=u \mathrm{~d} t,
$$

from (17) we easily obtain an exact dynamical equation (15). Moreover, one easily obtains that the dynamical mass, defined by means of expression (19), is given as

$$
m=m_{0}\left(1-u^{2}\right)^{-1 / 2},
$$

coinciding with the result (3) of the preceding section. Thereby, based on the above obtained results, one can formulate the following proposition.

Proposition 2.1 The alternative freely moving point particle electrodynamical model (15) allows the least action formulation (16) with respect to the "rest" reference system variables, where the Lagrangian function is given by expression (18). Its electrodynamics is completely equivalent to that of a classical relativistic freely moving point particle, described in section 2.

\subsection{A moving charged point particle - an alternative electrodynamical model}

Proceed now to the case where our charged point particle $q$ moves in the space time with velocity vector $u \in \mathbb{E}^{3}$ and interacts with another external charged point particle, moving with velocity vector $u_{\mathrm{f}} \in \mathbb{E}^{3}$ subject to some common reference system $\mathcal{K}$. As was shown in $[6,7]$, the corresponding dynamical equation on the vacuum potential field function $\bar{W}: \mathbb{M}^{4} \rightarrow \mathbb{R}$ is given as

$$
\frac{\mathrm{d}}{\mathrm{d} t}\left[-\bar{W}\left(u-u_{\mathrm{f}}\right)\right]=-\nabla \bar{W} .
$$

As the external charged particle moves in the space-time, it generates the related magnetic field $B:=\nabla \times A$, whose magnetic vector potential $A: \mathbb{M}^{4} \rightarrow \mathbb{E}^{3}$ is defined, owing to the results of $[6,7,18]$, as

$$
q A:=\bar{W} u_{\mathrm{f}} .
$$

Since, owing to (17), the particle momentum $p=-\bar{W} u$, equation (21) can be equivalently rewritten as

$$
\frac{\mathrm{d}}{\mathrm{d} t}(p+q A)=-\nabla \bar{W} .
$$

To represent the dynamical equation (23) within the classical Lagrangian formalism, we start from the following action functional naturally generalizing functional (16):

$$
S:=-\int_{\tau_{1}}^{\tau_{2}} \bar{W}\left(1+|\dot{r}-\dot{\xi}|^{2}\right)^{1 / 2} \mathrm{~d} \tau,
$$

where we denoted by $\dot{\xi}=u_{\mathrm{f}} \mathrm{d} t / \mathrm{d} \tau, \mathrm{d} \tau=\mathrm{d} t\left(1-\left|u-u_{\mathrm{f}}\right|^{2}\right)^{1 / 2}$, which take into account the relative velocity of our charged point particle $q$ with respect to the reference system $\mathcal{K}^{\prime}$, moving with velocity vector $u_{\mathrm{f}} \in \mathbb{E}^{3}$ subject to the reference system $\mathcal{K}$. In this case, evidently, our charged point particle $q$ moves with velocity vector $u-u_{\mathrm{f}} \in \mathbb{E}^{3}$ subject to the reference system $\mathcal{K}^{\prime}$, and the external charged particle is, respectively, in rest.

Compute now the least action variational condition $\delta S=0$, taking into account that, owing to (24), the corresponding Lagrangian function is given as

$$
\mathcal{L}:=-\bar{W}\left(1+|\dot{r}-\dot{\xi}|^{2}\right)^{1 / 2} .
$$

Thereby, the common particle momentum

$$
\begin{aligned}
P & :=\partial \mathcal{L} / \partial \dot{r}=-\bar{W}(\dot{r}-\dot{\xi})\left(1+|\dot{r}-\dot{\xi}|^{2}\right)^{-1 / 2} \\
& =-\bar{W} \dot{r}\left(1+|\dot{r}-\dot{\xi}|^{2}\right)^{-1 / 2}+\bar{W} \dot{\xi}\left(1+|\dot{r}-\dot{\xi}|^{2}\right)^{-1 / 2}=m u+q A:=p+q A
\end{aligned}
$$


and the dynamical equation is given as

$$
\frac{\mathrm{d}}{\mathrm{d} \tau}(p+q A)=-\nabla \bar{W}\left(1+|\dot{r}-\dot{\xi}|^{2}\right)^{1 / 2} .
$$

Taking into account that $\mathrm{d} \tau=\mathrm{d} t\left(1-\left|u-u_{\mathrm{f}}\right|^{2}\right)^{1 / 2}$ and $\left(1+|\dot{r}-\dot{\xi}|^{2}\right)^{1 / 2}=\left(1-\left|u-u_{\mathrm{f}}\right|^{2}\right)^{-1 / 2}$, we finally obtain from (27) exactly the dynamical equation (23). Thus, we can formulate our result as the next proposition.

Proposition 2.2 The alternative classical relativistic electrodynamical model (21) allows the least action formulation (24) with respect to the "rest" reference system variables, where the Lagrangian function is given by expression (25).

\subsection{A moving charged point particle - a dual to the classical alternative electrodynamical model}

It is easy to observe that the action functional (24) is written taking into account the classical Galilean transformations of reference systems. If we now consider the action functional (16) for a charged point particle, moving with respect the reference system $\mathcal{K}_{\mathrm{r}}$, and take into account its interaction with an external magnetic field, generated by the vector potential $A: \mathbb{M}^{4} \rightarrow \mathbb{E}^{3}$, it can be naturally generalized as

$$
S:=\int_{t_{1}}^{t_{2}}(-\bar{W} \mathrm{~d} t+q\langle A, \mathrm{~d} r\rangle)=\int_{\tau_{1}}^{\tau_{2}}\left[-\bar{W}\left(1+\dot{r}^{2}\right)^{1 / 2}+q\langle A, \dot{r}\rangle\right] \mathrm{d} \tau,
$$

where we accepted here that $\mathrm{d} \tau=\mathrm{d} t\left(1-u^{2}\right)^{1 / 2}$.

Thus, the corresponding common particle-field momentum looks as follows:

$$
P:=\partial \mathcal{L} / \partial \dot{r}=-\bar{W} \dot{r}\left(1+\dot{r}^{2}\right)^{-1 / 2}+q A=m u+q A:=p+q A,
$$

satisfying the equation

$$
\begin{aligned}
\dot{P} & :=\mathrm{d} P / \mathrm{d} \tau=\partial \mathcal{L} / \partial r=-\nabla \bar{W}\left(1+\dot{r}^{2}\right)^{1 / 2}+q \nabla\langle A, \dot{r}\rangle \\
& =-\nabla \bar{W}\left(1-u^{2}\right)^{-1 / 2}+q \nabla\langle A, u\rangle\left(1-u^{2}\right)^{-1 / 2}
\end{aligned}
$$

where

$$
\mathcal{L}:=-\bar{W}\left(1+\dot{r}^{2}\right)^{1 / 2}+q\langle A, \dot{r}\rangle
$$

is the corresponding Lagrangian function. Taking now into account that $\mathrm{d} \tau=\mathrm{d} t\left(1-u^{2}\right)^{1 / 2}$, one easily finds from (30) that

$$
\mathrm{d} P / \mathrm{d} t=-\nabla \bar{W}+q \nabla\langle A, u\rangle .
$$

Upon substituting (29) into (32) and making use of the well known [11] identity

$$
\nabla\langle a, b\rangle=\langle a, \nabla\rangle b+\langle b, \nabla\rangle a+b \times(\nabla \times a)+a \times(\nabla \times b),
$$

where $a, b \in \mathbb{E}^{3}$ are arbitrary vector functions, we finally obtain the classical expression for the Lorentz force $F$, acting on the moving charged point particle $q$ :

$$
\mathrm{d} p / \mathrm{d} t:=F=q E+q u \times B,
$$

where, by definition,

$$
E:=-\nabla \bar{W} q^{-1}-\partial A / \partial t
$$

is the corresponding electric field and

$$
B:=\nabla \times A
$$

is the corresponding magnetic field.

We formulate the obtained result as the next proposition. 
Proposition 2.3 The classical relativistic Lorentz force (34) allows the least action formulation (28) with respect to the "rest" reference system variables, where Lagrangian function is given by expression (31). Its electrodynamics, described by the Lorentz force (34) is completely equivalent to the classical relativistic moving point particle electrodynamics, described by means of the Lorentz force (12) in section 2.

Concerning the previously obtained dynamical equation (27) we can easily observe that it can be equivalently rewritten as follows:

$$
\mathrm{d} p / \mathrm{d} t=(-\nabla \bar{W}-q \mathrm{~d} A / \mathrm{d} t+q \nabla\langle A, u\rangle)-q \nabla\langle A, u\rangle .
$$

The latter, owing to (32) and (34), finally takes the following Lorentz type force form

$$
\mathrm{d} p / \mathrm{d} t=q E+q u \times B-q \nabla\langle A, u\rangle,
$$

found earlier in $[6,7,18]$.

Expressions (34) and (38) are equal to each other up to the gradient term $F_{\mathrm{c}}:=-q \nabla\langle A, u\rangle$, which allows to reconcile the Lorentz forces acting on a charged moving particle $q$ with respect to different reference systems. This fact is important for our vacuum field theory approach since it needs to use no special geometry and makes it possible to analyse both electromagnetic and gravitational fields simultaneously, based on a new definition of the dynamical mass by means of expression (19).

\section{The vacuum field theory electrodynamics equations: Hamiltonian analysis}

It is well know $[1,2,8,9,17]$ that any Lagrangian theory allows the equivalent canonical Hamiltonian representation via the classical Legendrian transformation. As we have already formulated above our vacuum field theory of a moving charged particle $q$ in the Lagrangian form, we proceed now to its Hamiltonian analysis making use of the action functionals (16), (25) and (28).

Take, first, the Lagrangian function (18) and the momentum expression (17) for defining the corresponding Hamiltonian function

$$
\begin{aligned}
H:=\langle p, \dot{r}\rangle-\mathcal{L} & =-\langle p, p\rangle \bar{W}^{-1}\left(1-p^{2} / \bar{W}^{2}\right)^{-1 / 2}+\bar{W}\left(1-p^{2} / \bar{W}^{2}\right)^{-1 / 2} \\
& =-p^{2} \bar{W}^{-1}\left(1-p^{2} / \bar{W}^{2}\right)^{-1 / 2}+\bar{W}^{2} \bar{W}^{-1}\left(1-p^{2} / \bar{W}^{2}\right)^{-1 / 2} \\
& =-\left(\bar{W}^{2}-p^{2}\right)\left(\bar{W}^{2}-p^{2}\right)^{-1 / 2}=-\left(\bar{W}^{2}-p^{2}\right)^{1 / 2} .
\end{aligned}
$$

As a result, we easily obtain $[1,2,8,9]$ that the Hamiltonian function (39) is a conservation law of the dynamical field equation (15), that is for all $\tau, t \in \mathbb{R}$

$$
\mathrm{d} H / \mathrm{d} t=0=\mathrm{d} H / \mathrm{d} \tau,
$$

which naturally allows to interpret it as the energy expression. Thus, we can write that the particle energy

$$
\mathcal{E}=\left(\bar{W}^{2}-p^{2}\right)^{1 / 2} .
$$

The suitable Hamiltonian equations, equivalent to the vacuum field equation (15), look as follows:

$$
\begin{aligned}
\dot{r} & :=\mathrm{d} r / \mathrm{d} \tau=\partial H / \partial p=p\left(\bar{W}^{2}-p^{2}\right)^{-1 / 2}, \\
\dot{p} & :=\mathrm{d} p / \mathrm{d} \tau=-\partial H / \partial r=\bar{W} \nabla \bar{W}\left(\bar{W}^{2}-p^{2}\right)^{-1 / 2} .
\end{aligned}
$$

Thereby, based on the above obtained results, one can formulate the following proposition.

Proposition 3.1 The alternative freely moving point particle electrodynamical model (15) allows the canonical Hamiltonian formulation (42) with respect to the "rest" reference system variables, where the Hamiltonian function is given by expression (39). Its electrodynamics is completely equivalent to the classical relativistic freely moving point particle electrodynamics, described in section 2. 
Based now on the Lagrangian expression (25) one can construct, the same way as above, the Hamiltonian function for the dynamical field equation (23), describing the motion of charged particle $q$ in external electromagnetic field in the canonical Hamiltonian form:

$$
\dot{r}:=\mathrm{d} r / \mathrm{d} \tau=\partial H / \partial P, \quad \dot{P}:=\mathrm{d} P / \mathrm{d} \tau=-\partial H / \partial r,
$$

where

$$
\begin{aligned}
H & :=\langle P, \dot{r}\rangle-\mathcal{L}=\left\langle P, \dot{\xi}-P \bar{W}^{-1}\left(1-P^{2} / \bar{W}^{2}\right)^{-1 / 2}\right\rangle+\bar{W}\left[\bar{W}^{2}\left(\bar{W}^{2}-P^{2}\right)^{-1}\right]^{1 / 2} \\
& =\langle P, \dot{\xi}\rangle+P^{2}\left(\bar{W}^{2}-P^{2}\right)^{-1 / 2}-\bar{W}^{2}\left(\bar{W}^{2}-P^{2}\right)^{-1 / 2}=-\left(\bar{W}^{2}-P^{2}\right)\left(\bar{W}^{2}-P^{2}\right)^{-1 / 2}+\langle P, \dot{\xi}\rangle \\
& =-\left(\bar{W}^{2}-P^{2}\right)^{1 / 2}-q\langle A, P\rangle\left(\bar{W}^{2}-P^{2}\right)^{-1 / 2}
\end{aligned}
$$

Here we took into account that, owing to definitions (22) and (26),

$$
\begin{aligned}
q A & :=\bar{W} u_{\mathrm{f}}=\bar{W} \mathrm{~d} \xi / \mathrm{d} t=\bar{W} \frac{\mathrm{d} \xi}{\mathrm{d} \tau} \cdot \frac{\mathrm{d} \tau}{\mathrm{d} t}=\bar{W} \dot{\xi}\left(1-|u-v|^{2}\right)^{1 / 2} \\
& =\bar{W} \dot{\xi}\left(1+|\dot{r}-\dot{\xi}|^{2}\right)^{-1 / 2}=-\bar{W} \dot{\xi}\left(\bar{W}^{2}-P^{2}\right)^{1 / 2} \bar{W}^{-1}=-\dot{\xi}\left(\bar{W}^{2}-P^{2}\right)^{1 / 2},
\end{aligned}
$$

or

$$
\dot{\xi}=-q A\left(\bar{W}^{2}-P^{2}\right)^{-1 / 2},
$$

where $A: \mathbb{M}^{4} \rightarrow \mathbb{R}^{3}$ is the related magnetic vector potential, generated by the moving external charged particle.

Thereby we can state that the Hamiltonian function (44) satisfies the energy conservation conditions

$$
\mathrm{d} H / \mathrm{d} t=0=\mathrm{d} H / \mathrm{d} \tau,
$$

for all $\tau, t \in \mathbb{R}$, that is the suitable energy expression

$$
\mathcal{E}=\left(\bar{W}^{2}-P^{2}\right)^{1 / 2}+q\langle A, P\rangle\left(\bar{W}^{2}-P^{2}\right)^{-1 / 2}
$$

holds. The result (48) essentially differs from that obtained in [11], which makes use of the well known Einsteinian Lagrangian for a moving charged point particle $q$ in external electromagnetic field. Thereby, our result can be formulated as follows.

Proposition 3.2 The alternative classical relativistic electrodynamical model (21) allows the Hamiltonian formulation (43) with respect to the "rest" reference system variables, where the Hamiltonian function is given by expression (44).

To make this difference more clear, below we will analyse the Lorentz force (34) from the Hamiltonian point of view based on the Lagrangian function (31). Thus, we obtain that the corresponding Hamiltonian function

$$
\begin{aligned}
H & :=\langle P, \dot{r}\rangle-\mathcal{L}=\langle P, \dot{r}\rangle+\bar{W}\left(1+\dot{r}^{2}\right)^{1 / 2}-q\langle A, \dot{r}\rangle=\langle P-q A, \dot{r}\rangle+\bar{W}\left(1+\dot{r}^{2}\right)^{1 / 2} \\
& =-\langle p, p\rangle \bar{W}^{-1}\left(1-p^{2} / \bar{W}^{2}\right)^{-1 / 2}+\bar{W}\left(1-p^{2} / \bar{W}^{2}\right)^{-1 / 2} \\
& =-\left(\bar{W}^{2}-p^{2}\right)\left(\bar{W}^{2}-p^{2}\right)^{-1 / 2}=-\left(\bar{W}^{2}-p^{2}\right)^{1 / 2} .
\end{aligned}
$$

Since $p=P-q A$, expression (49) takes the final "no interaction" [11,31,35,36] form

$$
H=-\left[\bar{W}^{2}-(P-q A)^{2}\right]^{1 / 2},
$$

being conservative with respect to the evolution equations (29) and (30), that is

$$
\mathrm{d} H / \mathrm{d} t=0=\mathrm{d} H / \mathrm{d} \tau
$$

for all $\tau, t \in \mathbb{R}$. The latter are simultaneously equivalent to the following Hamiltonian system:

$$
\begin{aligned}
\dot{r} & =\partial H / \partial P=(P-q A)\left[\bar{W}^{2}-(P-q A)^{2}\right]^{-1 / 2}, \\
\dot{P} & =-\partial H / \partial r=(\bar{W} \nabla \bar{W}-\nabla\langle q A,(P-q A)\rangle)\left[\bar{W}^{2}-(P-q A)^{2}\right]^{-1 / 2},
\end{aligned}
$$


which can be easily checked by direct calculations. Really, the first equation

$$
\begin{aligned}
\dot{r} & =(P-q A)\left[\bar{W}^{2}-(P-q A)^{2}\right]^{-1 / 2}=p\left(\bar{W}^{2}-p^{2}\right)^{-1 / 2} \\
& =m u\left(\bar{W}^{2}-p^{2}\right)^{-1 / 2}=-\bar{W} u\left(\bar{W}^{2}-p^{2}\right)^{-1 / 2}=u\left(1-u^{2}\right)^{-1 / 2}
\end{aligned}
$$

holds, owing to the condition $\mathrm{d} \tau=\mathrm{d} t\left(1-u^{2}\right)^{1 / 2}$ and definitions $p:=m u, m=-\bar{W}$, postulated from the very beginning. Similarly we obtain that

$$
\begin{aligned}
\dot{P} & =-\nabla \bar{W}\left(1-p^{2} / \bar{W}^{2}\right)^{-1 / 2}+\nabla\langle q A, u\rangle\left(1-p^{2} / \bar{W}^{2}\right)^{-1 / 2} \\
& =-\nabla \bar{W}\left(1-u^{2}\right)^{-1 / 2}+\nabla\langle q A, u\rangle\left(1-u^{2}\right)^{-1 / 2}
\end{aligned}
$$

exactly coinciding with equation (32) subject to the evolution parameter $t \in \mathbb{R}$. Our result we now formulate as the next proposition.

Proposition 3.3 The dual to the classical relativistic electrodynamical model (34) allows the Hamiltonian formulation (52) with respect to the "rest" reference system variables, where the Hamiltonian function is given by expression (50).

\section{The quantization of electrodynamics models within the vacuum field theory no-geometry approach}

\subsection{The problem setting}

In our recent works $[6,7]$ there was devised a new regular no-geometry approach to deriving from the first principles the electrodynamics of a moving charged point particle $q$ in external electromagnetic field. This approach has, in part, to reconcile the existing mass-energy controversy [14] within the classical relativistic electrodynamics. Based on the vacuum field theory approach proposed in $[6,7,18]$ we reanalysed this problem in the above sections both from Lagrangian and Hamiltonian points of view having derived crucial expressions for the corresponding energy functions and Lorentz type forces, acting on the moving charge point particle $q$.

Since all our electrodynamics models were represented here in the canonical Hamiltonian form, they are suitable for applying to them the Dirac type quantization procedure [10,3,13] and for regular obtaining the related Schrödinger type evolution equations. Namely, to this problem there is devoted this section.

\subsection{Free point particle electrodynamics model and its quantization}

The charged point particle electrodynamics models, discussed in detail in sections 2 and 3, were also considered in [7] from the dynamical point of view, where an attempt was made to apply the quantization Dirac type procedure to the corresponding conserved energy expressions. Nevertheless, within the canonical point of view, the true quantization procedure should be based on the suitable canonical Hamiltonian formulation of the models, which in the case under consideration looks as (42), (43) and (52).

In particular, consider a free charged point particle electrodynamics model, governed (42) by the following Hamiltonian equations:

$$
\mathrm{d} r / \mathrm{d} \tau:=\partial H / \partial p=-p\left(\bar{W}^{2}-p^{2}\right)^{-1 / 2}, \quad \mathrm{~d} p / \mathrm{d} \tau:=-\partial H / \partial r=-\bar{W} \nabla \bar{W}\left(\bar{W}^{2}-p^{2}\right)^{-1 / 2},
$$

where we denoted, as before, by $\bar{W}: \mathbb{M}^{4} \rightarrow \mathbb{R}$ the corresponding vacuum field potential, characterizing the medium field structure, by $(r, p) \in \mathbb{E}^{3} \times \mathbb{E}^{3}$ the standard canonical coordinatemomentum variables, by $\tau \in \mathbb{R}$ the proper "rest" reference system $\mathcal{K}_{\mathrm{r}}$ time parameter, related to our moving particle, and by $H: \mathbb{E}^{3} \times \mathbb{E}^{3} \rightarrow \mathbb{R}$ the Hamiltonian function

$$
H:=-\left(\bar{W}^{2}-p^{2}\right)^{1 / 2}
$$


expressed here and throughout further in the light speed units. The "rest" reference system $\mathcal{K}_{\mathrm{r}}$, parameterized by variables $(r, \tau) \in \mathbb{E}^{4}$, is related to any other reference system $\mathcal{K}$ subject to which our charged point particle $q$ moves with velocity vector $u \in \mathbb{E}^{3}$, and which is parameterized by variables $(r, t) \in \mathbb{M}^{4}$, via the following Euclidean infinitesimal relationship:

$$
\mathrm{d} t^{2}=\mathrm{d} \tau^{2}+\mathrm{d} r^{2}
$$

which is equivalent to the Minkovskian infinitesimal relationship

$$
\mathrm{d} \tau^{2}=\mathrm{d} t^{2}-\mathrm{d} r^{2}
$$

The Hamiltonian function (56) satisfies, evidently, the energy conservation conditions

$$
\mathrm{d} H / \mathrm{d} t=0=\mathrm{d} H / \mathrm{d} \tau
$$

for all $t, \tau \in \mathbb{R}$. This means that the suitable energy value

$$
\mathcal{E}=\left(\bar{W}^{2}-p^{2}\right)^{1 / 2}
$$

can be treated by means of the Dirac type quantization scheme [10] to obtain, as $\hbar \rightarrow 0$, (or the light speed $c \rightarrow \infty)$ the governing Schrödinger type dynamical equation. To do this, similarly to $[6,7]$, we need to make canonical operator replacements $\mathcal{E} \rightarrow \hat{\mathcal{E}}:=-\frac{\hbar}{\mathrm{i}} \frac{\partial}{\partial \tau}, p \rightarrow \hat{p}:=\frac{\hbar}{\mathrm{i}} \nabla$, as $\hbar \rightarrow 0$, in the following energy determining expression:

$$
\mathcal{E}^{2}:=(\hat{\mathcal{E}} \psi, \hat{\mathcal{E}} \psi)=\left(\psi, \hat{\mathcal{E}}^{2} \psi\right)=\left(\psi, \hat{H}^{+} \hat{H} \psi\right),
$$

where, by definition, owing to $(60)$,

$$
\hat{\mathcal{E}}^{2}=\bar{W}^{2}-\hat{p}^{2}=\hat{H}^{+} \hat{H}
$$

is a suitable operator factorization in the Hilbert space $\mathcal{H}:=L_{2}\left(\mathbb{R}^{3} ; \mathbb{C}\right)$ and $\psi \in \mathcal{H}$ is the corresponding normalized quantum vector state. Since the following elementary identity

$$
\bar{W}^{2}-\hat{p}^{2}=\bar{W}\left(1-\bar{W}^{-1} \hat{p}^{2} \bar{W}^{-1}\right)^{1 / 2}\left(1-\bar{W}^{-1} \hat{p}^{2} \bar{W}^{-1}\right)^{1 / 2} \bar{W}
$$

holds, we can put, by definition, following (62) and (63) that the operator

$$
\hat{H}:=\left(1-\bar{W}^{-1} \hat{p}^{2} \bar{W}^{-1}\right)^{1 / 2} \bar{W} .
$$

Having calculated the operator expression (64) as $\hbar \rightarrow 0$ up to the operator accuracy $O\left(\hbar^{4}\right)$, we can easily obtain

$$
\hat{H}=\frac{\hat{p}^{2}}{2 m(u)}+\bar{W}:=-\frac{\hbar^{2}}{2 m(u)} \nabla^{2}+\bar{W},
$$

where we took into account the dynamical mass definition $m(u):=-\bar{W}$ (in the light speed units). Thereby, based now on (61) and (65), we obtain, up to the operator accuracy $O\left(\hbar^{4}\right)$, the following Schrödinger type evolution equation

$$
\mathrm{i} \hbar \frac{\partial \psi}{\partial \tau}:=\hat{\mathcal{E}} \psi=\hat{H} \psi=-\frac{\hbar^{2}}{2 m(u)} \nabla^{2} \psi+\bar{W} \psi
$$

with respect to the "rest" reference system $\mathcal{K}_{\mathrm{r}}$ evolution parameter $\tau \in \mathbb{R}$. Concerning the related evolution parameter $t \in \mathbb{R}$, parameterizing a reference system $\mathcal{K}$, the equation (66) takes the following form:

$$
\mathrm{i} \hbar \frac{\partial \psi}{\partial t}=-\frac{\hbar^{2} m_{0}}{2 m(u)^{2}} \nabla^{2} \psi-m_{0} \psi
$$

Here we took into account that, owing to (60), the classical mass relationship

$$
m(u)=m_{0}\left(1-u^{2}\right)^{-1 / 2}
$$

holds, where $m_{0} \in \mathbb{R}_{+}$is the corresponding rest mass of our point particle $q$.

The obtained linear Schrödinger equation (67) for the case $\hbar / c \rightarrow 0$ really coincides with that well-known $[5,10,11]$ from classical quantum mechanics. 


\subsection{Classical charged point particle electrodynamics model and its quantization}

We start here from the first vacuum field theory reformulation of the classical charged point particle electrodynamics, considered in section 3 and based on the conserved Hamiltonian function $(50)$

$$
H:=-\left[\bar{W}^{2}-(P-q A)^{2}\right]^{1 / 2},
$$

where $q \in \mathbb{R}$ is the particle charge and $(\bar{W}, A) \in \mathbb{R} \times \mathbb{E}^{3}$ is the corresponding electromagnetic field potentials and $P \in \mathbb{E}^{3}$ is the common particle-field momentum, defined as

$$
P:=p+q A, \quad p:=m u,
$$

and satisfying the well known classical Lorentz force equation. Here $m:=-\bar{W}$ is the observable dynamical mass, related to our charged particle, $u \in \mathbb{E}^{3}$ is its velocity vector with respect to a chosen reference system $\mathcal{K}$, being all expressed here, as before, in the light speed units.

Since our electrodynamics, based on (69), is canonically Hamiltonian, the Dirac type quantization scheme

$$
P \rightarrow \hat{P}:=\frac{\hbar}{\mathrm{i}} \nabla, \quad \mathcal{E} \rightarrow \hat{\mathcal{E}}:=-\frac{\hbar}{\mathrm{i}} \frac{\partial}{\partial \tau}
$$

should be applied to the suitable energy expression

$$
\mathcal{E}:=\left[\bar{W}^{2}-(P-q A)^{2}\right]^{1 / 2}
$$

following from the conservation conditions

$$
\mathrm{d} H / \mathrm{d} t=0=\mathrm{d} H / \mathrm{d} \tau,
$$

satisfied for all $\tau, t \in \mathbb{R}$.

Doing now the same way as above, we can factorize the operator $\hat{E}^{2}$ as follows:

$$
\bar{W}^{2}-(\hat{P}-q A)^{2}=\bar{W}\left[1-\bar{W}^{-1}(\hat{P}-q A)^{2} \bar{W}^{-1}\right]^{1 / 2}\left[1-\bar{W}^{-1}(\hat{P}-q A)^{2} \bar{W}^{-1}\right]^{1 / 2} \bar{W}:=\hat{H}^{+} \hat{H},
$$

where, by definition, (here as $\hbar / c \rightarrow 0, \hbar c=$ const)

$$
\hat{H}:=\frac{1}{2 m(u)}\left(\frac{\hbar}{\mathrm{i}} \nabla-q A\right)^{2}+\bar{W}
$$

up to operator accuracy $O\left(\hbar^{4}\right)$. Thereby, the related Schrödinger type evolution equation in the Hilbert space $\mathcal{H}=L_{2}\left(\mathbb{R}^{3} ; \mathbb{C}\right)$ looks like

$$
\mathrm{i} \hbar \frac{\partial \psi}{\partial \tau}:=\hat{\mathcal{E}} \psi=\hat{H} \psi=\frac{1}{2 m(u)}\left(\frac{\hbar}{\mathrm{i}} \nabla-q A\right)^{2} \psi+\bar{W} \psi
$$

with respect to the rest reference system $\mathcal{K}_{\mathrm{r}}$ evolution parameter $\tau \in \mathbb{R}$. The corresponding Schrödinger type evolution equation with respect to the evolution parameter $t \in \mathbb{R}$ looks, respectively, like

$$
\mathrm{i} \hbar \frac{\partial \psi}{\partial t}=\frac{m_{0}}{2 m(u)^{2}}\left(\frac{\hbar}{\mathrm{i}} \nabla-q A\right)^{2} \psi-m_{0} \psi
$$

The Schrödinger type evolution equation $(75)($ as $\hbar / c \rightarrow 0, \hbar c=$ const) completely coincides $[10,12]$ with that well known from the classical quantum mechanics. 


\subsection{Modified charged point particle electrodynamics model and its quantization}

Consider now, from the canonical point of view of the true quantization procedure, the electrodynamics model, which looks like (27) and whose Hamiltonian function (44) is

$$
H:=-\left(\bar{W}^{2}-P^{2}\right)^{1 / 2}-q\langle A, P\rangle\left(\bar{W}^{2}-P^{2}\right)^{-1 / 2} .
$$

This means that the suitable energy function

$$
\mathcal{E}:=\left(\bar{W}^{2}-P^{2}\right)^{1 / 2}+q\langle A, P\rangle\left(\bar{W}^{2}-P^{2}\right)^{-1 / 2},
$$

where, as before,

$$
P:=p+q A, \quad p:=m u, \quad m:=-\dot{W},
$$

is a conserved quantity for (27), which we will canonically quantize via the Dirac procedure (71). To make this, let us consider the quantum condition

$$
\mathcal{E}^{2}:=(\hat{\mathcal{E}} \psi, \hat{\mathcal{E}} \psi)=\left(\psi, \hat{\mathcal{E}}^{2} \psi\right), \quad(\psi, \psi):=1,
$$

where, by definition, $\hat{\mathcal{E}}:=-\frac{\hbar}{\mathrm{i}} \frac{\partial}{\partial t}$ and $\psi \in \mathcal{H}=L_{2}\left(\mathbb{R}^{3} ; \mathbb{C}\right)$ is a suitable normalized quantum state vector. Making now use of the energy function (78), one can easily obtain that

$$
\mathcal{E}^{2}=\bar{W}^{2}-(P-q A)^{2}+q^{2}\left[\langle A, A\rangle+\langle A, P\rangle\left(\bar{W}^{2}-P^{2}\right)^{-1}\langle P, A\rangle\right]
$$

which upon the canonical Dirac type quantization $P \rightarrow \hat{P}:=\frac{\hbar}{\mathrm{i}} \nabla$ transforms into the symmetrized operator expression

$$
\hat{\mathcal{E}}^{2}=\bar{W}^{2}-(\hat{P}-q A)^{2}+q^{2}\langle A, A\rangle+q^{2}\langle A, \hat{P}\rangle\left(\bar{W}^{2}-\hat{P}^{2}\right)^{-1}\langle\hat{P}, A\rangle .
$$

Having factorized operator (82) in the form $\hat{\mathcal{E}}^{2}=\hat{H}^{+} \hat{H}$, we obtain that up to the operator accuracy $O\left(\hbar^{4}\right)($ as $\hbar / c \rightarrow 0, \hbar c=$ const $)$

$$
\hat{H}:=\frac{1}{2 m(u)}\left(\frac{\hbar}{\mathrm{i}} \nabla-q A\right)^{2}-\frac{q^{2}}{2 m(u)}\langle A, A\rangle-\frac{q^{2}}{2 m^{3}(u)}\left\langle A, \frac{\hbar}{\mathrm{i}} \nabla\right\rangle\left\langle\frac{\hbar}{\mathrm{i}} \nabla, A\right\rangle,
$$

where we put, as before, $m(u)=-\bar{W}$ in the light speed units. Thus, owing to (80) and (83), the resulting Schrödinger evolution equation takes the form

$$
\mathrm{i} \hbar \frac{\partial \psi}{\partial \tau}:=\hat{H} \psi=\frac{1}{2 m(u)}\left(\frac{\hbar}{\mathrm{i}} \nabla-q A\right)^{2} \psi-\frac{q^{2}}{2 m(u)}\langle A, A\rangle \psi-\frac{q^{2}}{2 m^{3}(u)}\left\langle A, \frac{\hbar}{\mathrm{i}} \nabla\right\rangle\left\langle\frac{\hbar}{\mathrm{i}} \nabla, A\right\rangle \psi
$$

with respect to the "rest" reference system proper evolution parameter $\tau \in \mathbb{R}$. Similarly one also obtains the related Schrödinger type evolution equation with respect to the time parameter $t \in \mathbb{R}$ which we will not deal here with. The result (84) essentially differs from the corresponding classical Schrödinger evolution equation (75) which, thereby, forces us to more thoroughly reanalyse the main physically motivated principles, put into the backgrounds of classical electrodynamical models, described by the Hamiltonian functions (69) and (77) giving rise to different Lorentz type force expressions. This analysis we are to do in detail in a next work under preparation.

\section{Conclusion}

Thereby, we can claim that all the dynamical field equations discussed above are canonical Hamiltonian systems with respect to the corresponding proper "rest" reference systems, parameterized by suitable time parameters $\tau \in \mathbb{R}$. Owing to the passing to the basic reference system $\mathcal{K}$ with the time parameter $t \in \mathbb{R}$ the related Hamiltonian structure is naturally lost, giving rise to a new interpretation of the real particle motion as the one having an absolute sense only with respect to the proper "rest" reference system and being completely relative with respect to all other 
reference systems. Concerning the Hamiltonian expressions (39), (44) and (50) obtained above, one observes that all of them strongly depend on the vacuum potential field function $\bar{W}: \mathbb{M}^{4} \rightarrow \mathbb{R}$, thereby dissolving the mass problem of the classical energy expression, pointed out earlier [14] by L. Brillouin. It is necessary to mention here that the procedure subject to the canonical Dirac type quantization can be applied only to the corresponding dynamical field systems considered with respect to their proper "rest" reference systems.

Remark 5.1 Some comments can be also made concerning the classical relativity principle. Namely, we have obtained our results without using any Lorentz transformations of reference systems but only the natural notion of the "rest" reference system and its suitable parametrization with respect to any other moving reference systems. It seems reasonable since, in reality, the true state changes of a moving charged particle $q$ are exactly realized only with respect to its proper "rest" reference system. Thereby, the only question, still left open here, is that about the physical justification of the corresponding relationship between time parameters of the moving and "rest" reference systems.

This relationship, being accepted throughout this work, looks like

$$
\mathrm{d} \tau=\mathrm{d} t\left(1-u^{2}\right)^{1 / 2}
$$

where $u:=\mathrm{d} r / \mathrm{d} t \in \mathbb{E}^{3}$ is the velocity vector with which the "rest" reference system $\mathcal{K}_{\mathrm{r}}$ moves with respect to another arbitrarily chosen reference system $\mathcal{K}$. The expression (85) means, in particular, that there holds the equality

$$
\mathrm{d} t^{2}-\mathrm{d} r^{2}=\mathrm{d} \tau^{2}
$$

which exactly coincides with the classical infinitesimal Lorentz invariant. Its appearance is, evidently, not casual here, since all our dynamical vacuum field equations were successively derived $[6,7]$ from the governing equations on the vacuum potential field function $W: \mathbb{M}^{4} \rightarrow \mathbb{R}$ in the form

$$
\partial^{2} W / \partial t^{2}-\nabla^{2} W=\rho, \quad \partial W / \partial t+\nabla(v W)=0, \quad \partial \rho / \partial t+\nabla(v \rho)=0
$$

being a priori Lorentz invariant, where we denoted the charge density by $\rho \in \mathbb{R}$ and the suitable local velocity of the vacuum field potential changes by $v:=\mathrm{d} r / \mathrm{d} t$. Thereby, the dynamical infinitesimal Lorentz invariant (86) reflects this intrinsic structure of equations (87). Being rewritten in the following nonstandard Euclidean form:

$$
\mathrm{d} t^{2}=\mathrm{d} \tau^{2}+\mathrm{d} r^{2}
$$

it gives rise to quite another time relationship between reference systems $\mathcal{K}$ and $\mathcal{K}_{\mathrm{r}}$ :

$$
\mathrm{d} t=\mathrm{d} \tau\left(1+\dot{r}^{2}\right)^{1 / 2}
$$

where, as earlier, we denoted by $\dot{r}:=\mathrm{d} r / \mathrm{d} \tau$ the related particle velocity with respect to the "rest" reference system. Thus, we observe that all our Lagrangian analysis completed in section 2 is based on the corresponding functional expressions written in these "Euclidean" space-time coordinates and with respect to which the least action principle was applied. So, we see that there exist two alternatives - the first is to apply the least action principle to the corresponding Lagrangian functions expressed in the Minkovski type space-time variables with respect to an arbitrarily chosen reference system $\mathcal{K}$, and the second is to apply the least action principle to the corresponding Lagrangian functions expressed in the space-time Euclidean type variables with respect to the "rest" reference system $\mathcal{K}_{\mathrm{r}}$.

A slightly amusing but exciting inference following from our analysis in this work is the fact that all the classical special relativity results related with electrodynamics of charged point particles can be obtained one-to-one making use of our new definitions of the dynamical particle mass and the least action principle with respect to the associated Euclidean type space-time variables parameterizing the "rest" reference system.

One more remark is appropriate here concerning the quantization procedure of the proposed electrodynamic models. If the dynamical vacuum field equations are expressed in the canonical 
Hamiltonian form, there remain only technical problems in order to quantize them and obtain the corresponding Schrödinger type evolution equations in suitable Hilbert spaces of quantum states. There exists still another important inference from the approach devised in this work, consisting in a complete loss of the essence of the well-known Einsteinian equivalence principle [4,5,11,26,31], which becomes superfluous for our vacuum field theory of electromagnetism and gravity.

Based on the canonical Hamiltonian formalism devised in this work, concerning the alternatively charged point particle electrodynamic models, we succeeded in treating their Dirac type quantization. The obtained results were compared with the classical ones, but the physically motivated choice of a true model is left for the future studies. Another important aspect of the developed vacuum field theory no-geometry approach to combining the electrodynamics with the gravity consists in singling out the decisive role of the related "rest" reference system $\mathcal{K}_{\mathrm{r}}$. Namely, with respect to the "rest" reference system evolution parameter $\tau \in \mathbb{R}$, all our electrodynamic models make both the Lagrangian and Hamiltonian formulations suitable for the canonical quantization. The physical nature of this phenomenon remains insufficiently understandable so far. There is $[11,26,29,31,30]$ no physically reasonable explanation of this decisive role of the "rest" reference system, except for the very interesting reasonings by R. Feynman who argued in [5] that the relativistic expression for the classical Lorentz force (12) has physical sense only with respect to the "rest" reference system variables $(r, \tau) \in \mathbb{E}^{4}$. In the sequel of our work we are to analyse the quantization scheme more in detail and make a step toward the vacuum quantum field theory of infinite many particle systems.

\section{Acknowledgements}

The Authors are cordially thankful to the Abdus Salam International Centre for Theoretical Physics in Trieste, Italy, for the hospitality during their research 2007-2008 scholarships, and to the Institute for Condensed Matter Physics for the invitation to take part in the International Conference "Statistical Physics" held in Lviv, Ukraine, during 23-30 June 2009. A. P. is, especially, grateful to Profs. P.I. Holod (Kyiv, UKMA), J.M. Stakhira (Lviv, NUL), U. Taneri (Cyprus, EMU), J. Sławianowski (Warsaw, IPPT), Z. Peradzyński (Warsaw, UW) and M. Błaszak (Poznań, UP) for fruitful discussions, useful comments and remarks. Last but not least thanks go to academician Prof. A.A. Logunov for his interest to the work, as well as to Mrs. Dilys Grilli (Trieste, Publications office, ICTP) and Natalia K. Prykarpatska for professional help in preparing the manuscript for publication.

\section{References}

1. Arnold V.I. Mathematical Methods of Classical Mechanics. Springer, New York, 1978.

2. Abraham R., Marsden J. Foundation of Mechanics. The Benjamin/Cummings Publ. Co, Masachusets, 1978.

3. Bogoliubov N.N., Shirkov D.V. Quantum Fields. Nauka, Moscow, 1984.

4. Feynman R.P.M. Lectures on Gravitation. Notes of California Inst. of Technology, 1971.

5. Feynman R., Leighton R., Sands M. The Feynman Lectures on Physics. Electrodynamics, vol. 2. Addison-Wesley Publ. Co., Massachusetts, 1964.

6. Prykarpatsky A.K., Bogolubov N.N. (Jr.), Taneri U. Theor. Math. Phys., 2009, 160, 1079-1095.

7. Prykarpatsky A.K., Bogolubov N.N. (Jr.), Taneri U. Preprint ICTP, Trieste, IC/2008/051 (http://publications.ictp.it).

8. Prykarpatsky A., Mykytiuk I. Algebraic Integrability of Nonlinear Dynamical Systems Onmanifolds: Classical and Quantum Aspects. Kluwer Academic Publishers, Netherlands, 1998.

9. Thirring W. Classical Mathematical Physics. Third Edition. Springer, 1992.

10. Dirac P.A.M. The Principles of Quantum Mechanics. Second edition. Clarendon Press, Oxford, 1935.

11. Landau L.D., Lifshitz E.M. Field Theory, vol. 2. Nauka, Moscow, 1973.

12. Landau L.D., Lifshitz E.M. Quantum Mechanics, vol. 6. Nauka, Moscow, 1974.

13. Bogolubov N., Shirkov D. Introduction to the Theory of Quantized Fields. Interscience, New York, 1959.

14. Brillouin L. Relativity reexamined. Academic Press Publ., New York and London, 1970.

15. Faddeev L.D. Russian Physical Surveys, 1982, 136, No. 3, 435-457 (in Russian). 
16. Marsden J., Chorin A. Mathematical Foundations of the Mechanics of Liquid. Springer, New York, 1993.

17. Holod P.I., Klimyk A.I. Mathematical Foundations of Symmetry Theory. Naukova Dumka, Kyiv, 1992 (in Ukrainian).

18. Repchenko O. Field Physics. Galeria Publ., Moscow, 2005.

19. Brans C.H., Dicke R.H. Phys. Rev., 1961, 124, 925.

20. Deser S., Jackiw R. Preprint arXiv:hep-th/9206094, 1992.

21. Dunner G., Jackiw R. Preprint arXiv:hep-th/92004057, 1992.

22. Jackiw R., Polychronakos A.P. Preprint arXiv:hep-th/9809123, 1998.

23. Wilchek F. Ann. Henry Poincare, 2003, 4, 211-228.

24. Bialynicky-Birula I. Phys. Rev., 1967, 155, 1414; Bialynicky-Birula I. Phys. Rev., 1968, 166, 1505.

25. Kleinert H. Path Integrals. Second Edition, p. 685. World Scientific, 1995.

26. Klymyshyn I.A. Relativistic Astronomy. Naukova Dumka, Kyiv, 1980 (in Ukrainian).

27. Logunov A.A., Mestvirishvili M.A. Relativistic Theory of Gravitation. Nauka, Moscow, 1989 (in Russian).

28. Logunov A.A. The Theory of Gravity. Nauka, Moscow, 2000.

29. Logunov A.A. Relativistic Theory of Gravitation. Nauka, Moscow, 2006 (in Russian).

30. Logunov A.A. Lectures on Relativity Theory and Gravitation. Nauka, Moscow, 1987.

31. Pauli W. Theory of Relativity. Oxford Publ., Oxford, 1958.

32. Weinstock R. Am. J. Phys., 1965, 33, 640-645.

33. Mermin N.D. Am. J. Phys., 1984, 52, 119-124.

34. Mermin N.D. It's About Time: Understanding Einstein's Relativity. Princeton University Press, Princeton, NJ., 2005.

35. Kupershmidt B.A. Diff. Geom. \& Appl., 1992, 2, 275-293.

36. Prykarpatsky Y.A., Samoilenko A.M., Prykarpatsky A.K. Opuscula Mathematica, 2005, 25, No. 2, 287-298.

37. Green B. The Fabric of the Cosmos. Vintage Books Inc., New York, 2004.

38. Newman R.P. Comm. Mathem. Physics, 1989, 123, 17-52.

\title{
Лагранжів та Гамільтонів аналіз деяких релятивістичних моделей електродинаміки та їх квантування
}

\author{
М.М. Боголюбов (мол.) $)^{1,2}$, А.К. Прикарпатський \\ ${ }^{1}$ Математичний інститут ім. В.А. Стєклова РАН, Москва, Російська Федерація \\ 2 Міжнародний центр теоретичної фізики ім. Абдуса Салама, Трієст, Італія \\ 3 Гірничо-Металургійна академія ім. Ст. Сташіца, 30-059 Краків, Польща \\ 4 Державний педагогічний університет ім. І. Франка, Дрогобич, Україна
}

Отримано 19 червня 2009 р., в остаточному вигляді - 15 липня 2009 р.

Робота присвячена вивченню лагранжевих та гамільтонових властивостей деяких релятивістичних моделей електродинаміки і продовжує наші попередні дослідження. Базуючись на теоретикопольовому підході, розроблено Лагранжеве та Гамільтонове формулювання деяких класичних моделей електродинаміки. Розвинуто процедуру квантування типу Дірка, що базується на класичному гамільтоновому формулюванні. В рамках запропонованого підходу аналізується можливість комбінованого опису електродинаміки і гравітації.

Ключові слова: Лагранжів і Гамільтонів формалізм, релятивістичні моделі електродинаміки, квантування типу Дірака

PACS: $11.10 . W x, 05.30 .-d$ 